\title{
Accuracy of multiparametric magnetic resonance imaging for diagnosing prostate Cancer: a systematic review and meta- analysis
}

Liang Zhen ${ }^{1}$, Xiaoqiang Liu ${ }^{1 *}$, Chen Yegang ${ }^{2}$, Yang Yongjiao ${ }^{1}$, Xu Yawei ${ }^{1}$, Kang Jiaqi ${ }^{1}$, Wang Xianhao ${ }^{1}$, Song Yuxuan ${ }^{1}$, Hu Rui ${ }^{1}$, Zhang Wei ${ }^{1}$ and Ou Ningjing ${ }^{1}$

\begin{abstract}
Background: The application of multiparametric magnetic resonance imaging (mpMRI) for diagnosis of prostate cancer has been recommended by the European Association of Urology (EAU), National Comprehensive Cancer Network (NCCN), and European Society of Urogenital Radiology (ESUR) guidelines. The purpose of this study is to systematically review the literature on assessing the accuracy of mpMRI in patients with suspicion of prostate cancer.
\end{abstract}

Method: We searched Embase, Pubmed and Cochrane online databases from January 12,000 to October 272,018 to extract articles exploring the possibilities that the pre-biopsy mpMRI can enhance the diagnosis accuracy of prostate cancer. The numbers of true- and false-negative results and true- and false-positive ones were extracted to calculate the corresponding sensitivity and specificity of mpMRI. Study quality was assessed using QUADAS-2 tool. Random effects meta-analysis and a hierarchical summary receiver operating characteristic (HSROC) plot were performed for further study.

Results: After searching, we acquired 3741 articles for reference, of which 29 studies with 8503 participants were eligible for inclusion. MpMRI maintained impressive diagnostic value, the area under the HSROC curve was 0.87 (95\% $\mathrm{Cl}, 0.84-0.90)$. The sensitivity and specificity for mpMRI were $0.87[95 \% \mathrm{Cl}, 0.81-0.91]$ and $0.68[95 \% \mathrm{Cl}, 0.56-0.79]$ respectively. The positive likelihood ratio was 2.73 [95\%Cl 1.90-3.90]; negative likelihood ratio was 0.19 [95\% Cl 0.14,$0.27]$. The risk of publication bias was negligible with $P=0.96$.

Conclusion: Results of the meta-analysis suggest that mpMRl is a sensitive tool to diagnose prostate cancer. However, because of the high heterogeneity existing among the included studies, further studies are needed to apply the results of this meta-analysis in clinic.

\section{Background}

Prostate cancer $(\mathrm{PCa})$ is the most commonly diagnosed disease for male around the world [1]. The patients diagnosed by 2012 were 1.1 million, which accounted for $15 \%$ of the malignancy tumor, and its incidence and mortality have been increasing [2]. The current standard of diagnosing $\mathrm{PCa}$ in suspicious men depends on

\footnotetext{
* Correspondence: 847377491@qq.com

'Department of Urology, Tianjin Medical University General Hospital, Tianjin 300211, People's Republic of China

Full list of author information is available at the end of the article
}

transrectal ultrasound (TRUS)-guided prostate biopsy test which contains: transrectal ultrasound guided systematic biopsy; transperineal template biopsy and other method guided by transrectal ultrasound without knowing the exact location of cancer. According to a recent prospective study, the sensitivity of TRUS-biopsy to diagnose $\mathrm{PCa}$ is only $70.4 \%$ [3]. With the improvement in technology and the progress of modern medicine, patients have higher expectations for the prognosis. Multiparametric magnetic resonance imaging (mpMRI) has been increasingly used for guiding several aspects of

(c) The Author(s). 2019 Open Access This article is distributed under the terms of the Creative Commons Attribution 4.0 International License (http://creativecommons.org/licenses/by/4.0/) which permits unrestricted use, distribution, and reproduction in any medium, provided you give appropriate credit to the original author(s) and the source, provide a link to the Creative Commons license, and indicate if changes were made. The Creative Commons Public Domain Dedication waiver (http://creativecommons.org/publicdomain/zero/1.0/) applies to the data made available in this article, unless otherwise stated. 
prostate cancer management, including detection, staging, and treatment. The established parameters of mpMRI included T2-weighted images (T2WI), diffusion-weighted imaging (DWI), dynamic contrast-enhancement (DCE), and MR spectroscopy [4]. Abundant evidence based literature has shown that the pre-biopsy mpMRI can be used to improve the diagnosis accuracy of prostate cancer. MRI can provide internal zonal anatomy of the prostate and its extraprostatic extension of tumor, which can improve functional assessment and tumor grading in clinic [5]. The increasingly better resolution with the best depiction of prostate contours could also facilitate tumor localization [6]. In a addition, Yerram et al. [7] indicated that a low suspicion lesions on mpMRI has been shown to have a reliable negative predictive value (NPV) (90-98\%) for either low-grade tumors or negative biopsies that are suitable for active surveillance. Two systematic meta-analyses which explored the role of mpMRI in PCa have been published recently. In the study by Hamoen et al. [8] which evaluated 14 studies, its pooled sensitivity and specificity were 0.81 (95\% CI 0.76-0.85) and 0.77 (95\% CI 0.68-0.86), respectively. In a more recent meta-analysis by Moldovan et al. [9] the pooled NPV for overall prostate cancer was $82.4 \%$ (interquartile range IQR, $69.0-92.4 \%$ ) and $88.1 \%$ (IQR, 85.7-92.3) for clinical significant prostate cancer (csPCa) respectively.

However results of mpMRI may vary sharply because of difficulties in interpretation, lacking of standardized criteria for positive definition and the ability of radiologists [10]. Besides, the disadvantages of mpMRI such as equipment-specialization and time-consuming also impede its wide application [11]. Therefore, the routine application of mpMRI is still a topic of controversy due to the high variability among studies evaluating the diagnostic accuracy of mpMRI in staging and prediction of prostate cancer [12].

Some authors believed that mpMRI could be used as a preliminary screening so that some unnecessary prostate biopsies could be avoided even for the patients in active surveilance [13]. However, evidences to support such viewpoint are not convincing enough and it depends upon the sensitivity and specificity of mpMRI $[14,15]$ which would vary due to different MRI protocol, standard reference, and study quality. Ivo et al. $[14,15]$ indicated that whether patients with negative mpMRI findings could obviate biopsy remained highly controversial and it was still premature to draw a definite conclusion. Aydin et al. [16] indicated both highly vascularized benign prostatic hyperplasia nodules and prostatitis could lead to increased vessel enhancement in DCE imaging, which may cause low specificity of mpMRI. The selection bias of the two previous meta-analysis were of high level of heterogeneity because they did not exclude retrospectively designed study, besides, subgroup analysis was not performed specifically. Therefore the purpose of this study is to perform a systematic review and meta-analysis to estimate the diagnostic performance of mpMRI for detecting prostate cancer.

\section{Method}

A systematic review was conducted under the guidance of the Preferred Reported Items for Systematic Reviews and Meta-Analysis (PRISMA) [17]. PubMed, Embase and Cochrane online databases were searched from January 12,000 to October 272,018 to select qualified studies evaluating the diagnosis accuracy of mpMRI for the detection of prostate cancer. The search string combined synonyms of prostate cancer, MRI as follows: (prostate cancer OR prostatic cancer OR prostate neoplasm OR prostatic neoplasm OR prostate tumor OR prostatic tumor OR prostate (magnetic e resonance imaging OR MRI OR MR) carcinoma OR prostatic carcinoma OR prostate cancer). We included all original studies if they satisfied all the following requirements.

I. Studies should be prospectively designed without subjects-selection bias.

II. The available data is sufficient enough to calculate the diagnostic sensitivity and specificity of mpMRI.

III. The pathology results were provided by prostatectomy or prostate biopsy as reference to verify the mPMRI diagnose.

IV. Sufficient data of at least 10 patients is provided to construct $2 \times 2$ contingency tables.

V. The enrolled patients underwent T2WI and at least one functional imaging technique such as DWI, DCEI, or magnetic resonance spectroscopic imaging (MRSI). The Quality Assessment of Diagnostic Accuracy Studies 2 (QUADAS-2) tool was used to ascertain the quality of studies and likelihood of bias. The included articles were evaluated from the following aspects to decide if they were eligible for further analysis: index test (Describe the index test and how it was conducted and interpreted), patient selection (Describe methods of patient selection), flow and timing (Describe the interval and any interventions between index tests and the reference standard), reference standard (Describe the reference standard and how it was conducted and interpreted), as well as the concerns for applicability. Studies with high risk bias in more than two indexes would be excluded from our research. Only the studies involving biopsy-naive patients and/or patients with a history of the negative biopsy were adopted, and there was no restriction on the biopsy technique or the number of biopsies. 
Two reviewers (LZ and YY) independently checked titles and abstracts of all retrieved articles and determined final eligibility according to the previously mentioned criteria. Any disagreements between reviewers required consensus or references of a third reviewer (LX). All screening was performed by a pre-specified data extraction form.

The meta-analysis was carried out through a random effects model in RevMan 5.3. Measures such as diagnostic accuracy, including area under the receiver operating characteristic curve (AUC), sensitivity, specificity, positive predictive value and negative predictive value were calculated with corresponding 95\% CIs [18]. Data were analyzed with Review Manager 5 and Meta-Disc.

Multivariate meta-regression for sensitivity and specificity were applied to explore the possible source of heterogeneity. The factors that may have an impact on performance of mpMRI such as patient enrollment (consecutive versus not consecutive), reference standard (high risk of bias versus low risk of bias), whether the readers were blinded to histologic findings (blinded versus not blinded), the application of endorectal coil (ERC) (applied versus not applied), MRI field strength ( $3 \mathrm{~T}$ versus $1.5 \mathrm{~T}$ ), whether DCEI was performed (mutiparametric versus biparametric) were introduced as variables into the meta-regression where $P<0.05$ indicated a contribution to heterogeneity. The subgroup metaregression was based on data from the main analysis.

Heterogeneity was assessed using the $\mathrm{I}^{2}$ statistical method, with $\mathrm{I}^{2}>50 \%$ or $P$ value $<0.05$ indicating significant heterogeneity. Deeks' analysis was performed to evaluate the publication bias, with $P<0.05$ suggesting publication bias [19].

\section{Results}

Study selection

The study selection process and reasons for exclusion were depicted in the flow diagram [Fig. 1]. A total number of 3741 citations were initially identified. After abstract screening and removal of duplicates, 325 studies were selected for detailed evaluation. At last, 29 studies met all eligibility criteria $[3,15,20-46]$.

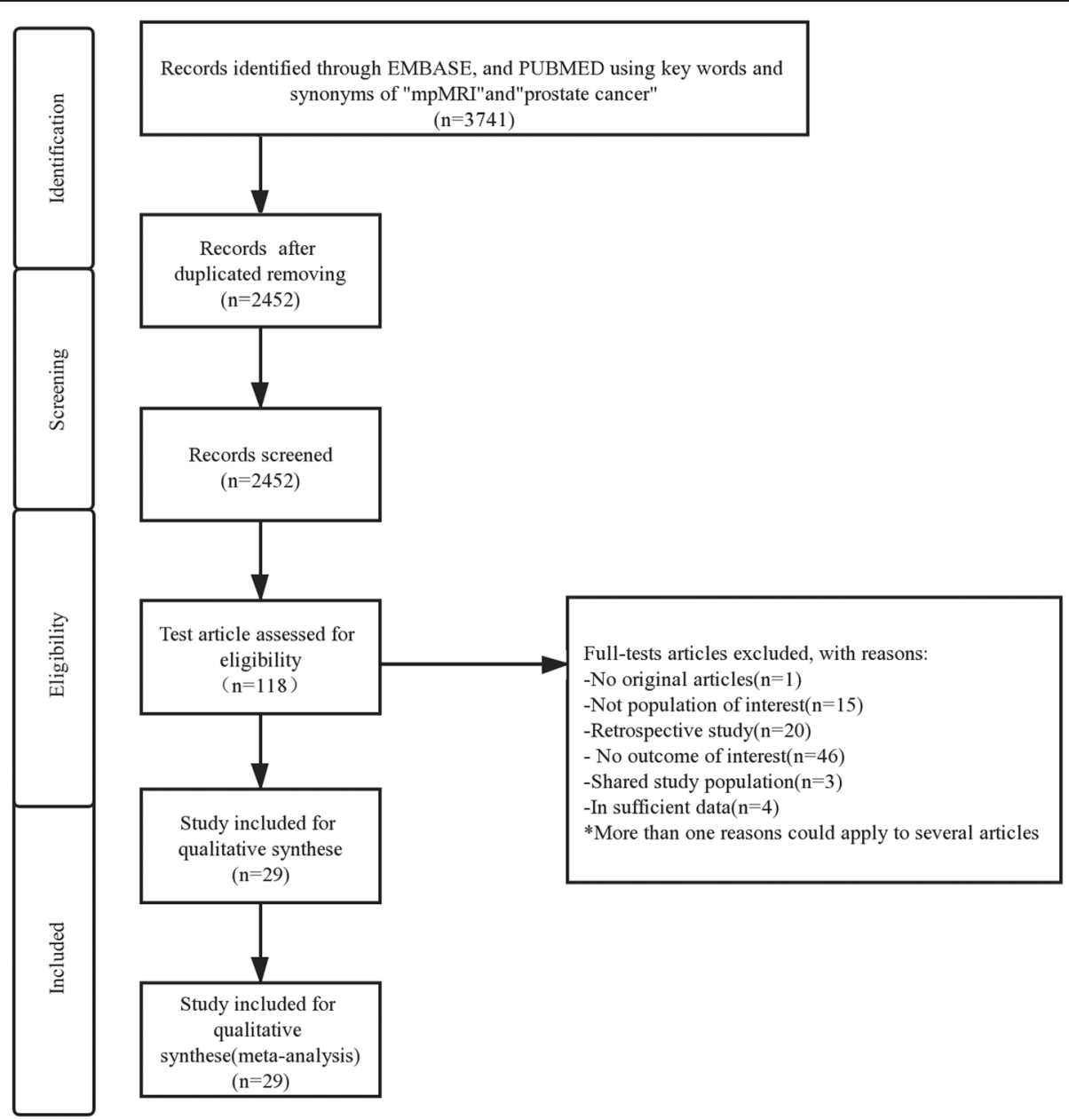

Fig. 1 Preferred reporting items for systematic reviews and Meta-analysis flow chart 


\section{Characteristics of included studies}

Eight thousand five hundred three who underwent prostate pre-biopsy mpMRI were involved in the 29 studies. The population size of study varied from 26 to 1448 patients. The involved 29 cohorts were carried out in the China, Egypt, Romania, Switzerland, Australia, France, USA, UK, Japan, Germany, Italy and India. The publication time ranged from 2007 to 2017. The study and patient elementary characteristics were displayed in Table 1. The target patients were biopsy-naive men in 8 studies $[3,15,20,22,23,37,38,41]$, and men with at least one previous negative biopsy in 8 studies $[21,25,31,34,39,42,44,45]$; in 9 studies $[26,29,30,32,33,35,40,43,46]$ the biopsy history of the patients was unclear. In rest studies patients were both biopsy-naive men and men with a history of previous negative biopsy. The age range of men was from 26 to 91 years (with an average of 65.8) and the PSA value ranged from $0.02-9796 \mathrm{ng} / \mathrm{ml}$.

The magnetic field strength was $1.5 \mathrm{~T}$ in 17 studies $[20,21,23,25,26,30-32,37-42,44,46,47]$ and $3 \mathrm{~T}$ in 10 studies [3, 15, 22, 24, 27, 34-36, 43, 45], respectively. DWI was conducted in 26 studies [3, 15, 20-29, 31-40, 42-44] and DCEI was conducted in 20 studies [3, 15, 21-29, 33$37,39,42-44]$, respectively. 10 studies $[15,20,23,25,30$, 32, 34, 35, 45, 46] also adopted MRSI. An ERC was used in 9 studies $[15,20,21,24,29,32-34,44]$. The definition of positive mpMRI was different from studies. Prostate Imaging Reporting Data System (PI-RADS) score system was used in 11 studies [3, 15, 21-28, 31, 33]. The reference standard was based on radical prostatectomy (RP) in 1 studies [22], TRUS-guided systematic biopsy in 22 studies [3, 20, 21, 23-27, 29, 30, 32-36, 38-44, 46], MRITRUS fusion-guided targeted biopsy or MRI-guided biopsy in 12 studies [3, 25-27, 31, 34, 36, 39-41, 45, 46]., transperineal template saturation biopsy in 3 studies [15, 28 , 37]. Some studies used the combination of these standards. The per-patient analysis was adopted in 24 studies [3, 20-22, 24-29, 31-38, 40, 41, 43-46], others were analyzed by lesion or lobe.

\section{Sensitivity and specificity of pre-biopsy mpMRI}

The diagnostic performance of prebiopsy multiparametric MRI of each included studies were demonstrated in Table 2. The sensitivity of mpMRI ranged from 42 to $100 \%$, and the specificity ranged from 12 to $100 \%$. The pooled sensitivity was 0.87 [95\% CI (0.81-0.91)] with heterogeneity $\left(\mathrm{I}^{2}=95.48 \mathrm{P}<0.01\right)$ and a pooled specificity of 0.68 [95\% CI $(0.56-0.79)]$ with heterogeneity $\left(\mathrm{I}^{2}=97.40, P<0.01\right)$ [Fig. 2]. At the patient level, the median biopsy positive rate was $49 \%$. The area under the hierarchical summary receiver operating characteristic curve (HSROC) was 0.87 (95\%CI, 0.84-0.90) [Fig. 3]; positive likelihood ratio was 2.73 [95\%CI 1.90-3.90]; negative likelihood ratio was 0.20 (95\%CI, 0.14,-0.27); and diagnostic odds ratio (DOR) was 14.00 (95\%CI, 7.88-24.84). The median mpMRI NPV was 0.79 (IQR, $0.70-0.92)$.

\section{Subgroup analysis}

According to our subgroup analysis, many factors, such as patient enrollment (consecutive versus not consecutive), reference standard (high risk of bias versus low risk of bias), whether the readers were blinded to histologic findings (blinded versus not blinded), the application of ERC, and MRI field strength (3 $\mathrm{T}$ versus $1.5 \mathrm{~T}$ ) showed significant independence associated with sensitivity $(P<$ 0.05 for all). Moreover, these previously mentioned factors affected only sensitivity, and none of those factors has an impact on specificity. As for the subgroup exploring the application of DCEI, there is no significant difference between two groups in both sensitivity and specificity. The results of our subgroup analysis were shown in Table 3.

\section{Quality of studies}

Regarding the patient selection domain, 7 studies $[15,22$, 29-31, 35, 46] had a high risk of bias because consecutive enrollment was not used or did not mention the exclusion criteria. Regarding the index test domain, in 10 studies $[15,25,26,30,32-35,44,46]$ the cut-off value for determining PCa was not specifically prior to interpretation. 6 studies [30, 31, 38, 40, 41, 46] did not have complete MRI parameters. Regarding the reference standard domain, we considered RP and targeted biopsy as the low risk reference standard. The TRUS-guided systematic biopsy or transperineal saturartion biopsy were considered to have a high risk of bias. Therefore, the risk of bias regarding the reference standard was high in 12 studies [3, 15, 24-27, 33, 34, 38, 40, $41,45]$. In 13 included studies, [3, 15, 27-29, 31, 32, $37,38,42,44,45,47]$ the blinding method was applied; however, other 16 studies did not explicitly mention blind method. There was no high risk of bias in any of the included studies in flow and timing domain [Fig. 4a and b]. The Higgins $\mathrm{I}^{2}$ statistics illustrated remarkable heterogeneity in terms of the sensitivity $\left(\mathrm{I}^{2}=95.48 \%\right)$ and specificity $\left(\mathrm{I}^{2}=97.40 \%\right)$. According to the Deeks' funnel plot, the likelihood of publication bias was low, with a $p$ value of 0.96 for the slope coefficient.[Fig. 5].

\section{Discussion}

In our meta-analysis, we assessed the diagnostic accuracy of mpMRI for detecting PCa. The results of our meta-analysis showed that the diagnostic accuracy of mpMRI for detecting $\mathrm{PCa}$ in 29 included studies was 


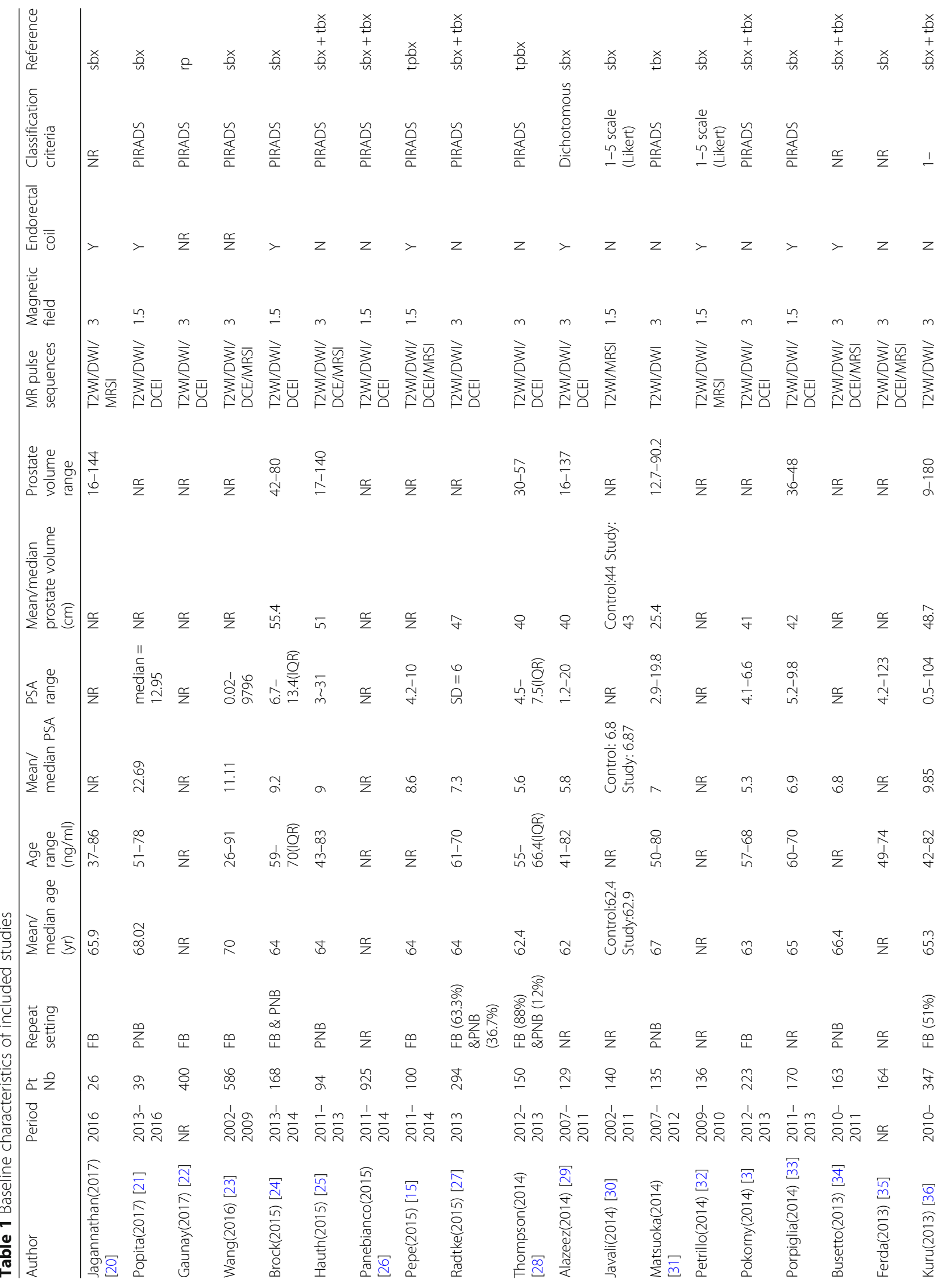


Zhen et al. BMC Cancer $\quad$ (2019) 19:1244

Page 6 of 15

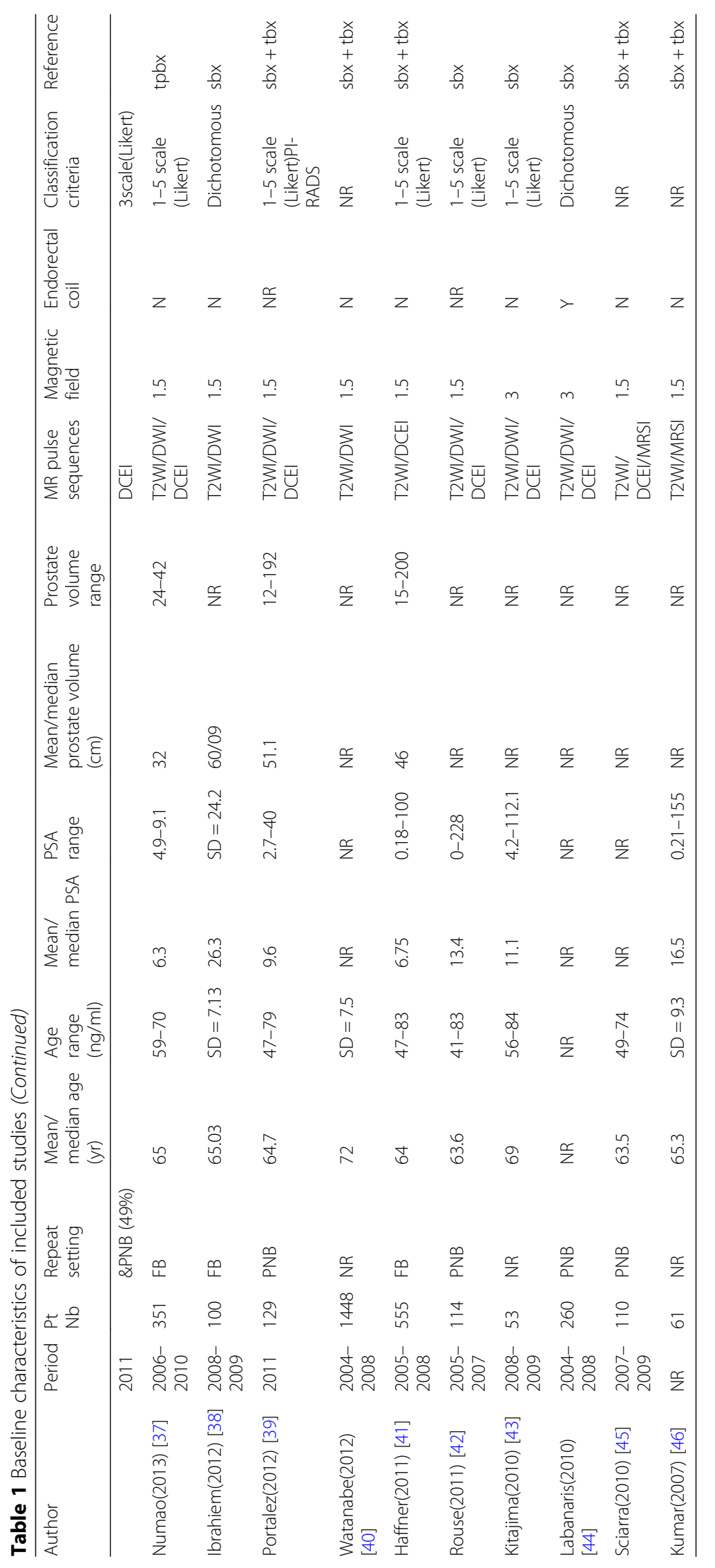


Table 2 Diagnostic performance of prebiopsy multiparametric MRI using biopsy findings as reference

\begin{tabular}{|c|c|c|c|c|c|c|c|c|c|}
\hline Author & Prevalence & Reporting Level & Tn & $\mathrm{Fn}$ & $T p$ & $\mathrm{Fp}$ & Sensitivity & Specificity & NPV \\
\hline Jagannathan(2017) & 0.73 & Patient & 6 & 6 & 17 & 1 & 0.89 & 0.86 & 0.50 \\
\hline Popita(2017) & 0.57 & Patient & 19 & 0 & 16 & 4 & 1.00 & 0.83 & 1.00 \\
\hline Gaunry(2016) & 0.48 & Patient & 41 & 24 & 376 & 72 & 0.94 & 0.36 & 0.63 \\
\hline Brock(2015) & 0.58 & Patient & 17 & 7 & 56 & 88 & 0.89 & 0.16 & 0.7 \\
\hline Hauth(2015) & 0.42 & Patient & 6 & 1 & 42 & 45 & 0.98 & 0.12 & 0.86 \\
\hline Panebianco(2015) & 0.45 & Patient & 104 & 43 & 186 & 22 & 0.81 & 0.83 & 0.7 \\
\hline Pepe(2015) & 0.54 & Lobe & 24 & 6 & 49 & 4 & 0.89 & 0.86 & 0.80 \\
\hline Radtke(2015) & 0.51 & Patient & 138 & 78 & 72 & 6 & 0.48 & 0.96 & 0.63 \\
\hline Thompson(2015) & 0.61 & Patient & 74 & 6 & 137 & 127 & 0.96 & 0.37 & 0.92 \\
\hline Wang(2015) & 1.00 & Patient & 200 & 8 & 332 & 53 & 0.98 & 0.79 & 0.96 \\
\hline Alazeez(2014) & 0.16 & Patient & 33 & 14 & 127 & 84 & 0.90 & 0.28 & 0.70 \\
\hline Javali(2014) & 0.64 & Lobe & 49 & 1 & 22 & 68 & 0.96 & 0.42 & 0.98 \\
\hline Matsuoka(2014) & 0.37 & Patient & 46 & 49 & 149 & 26 & 0.75 & 0.64 & 0.48 \\
\hline Petrillo(2014) & 0.18 & Patient & 56 & 4 & 21 & 55 & 0.84 & 0.50 & 0.93 \\
\hline Pokorny(2014) & 0.63 & Patient & 56 & 25 & 101 & 0 & 0.80 & 1.00 & 0.69 \\
\hline Porpiglia(2014) & 0.30 & Patient & 107 & 5 & 47 & 11 & 0.90 & 0.91 & 0.95 \\
\hline Busetto(2013) & 0.41 & Patient & 59 & 7 & 61 & 36 & 0.90 & 0.62 & 0.89 \\
\hline Ferda(2013) & 0.51 & Patient & 52 & 2 & 82 & 28 & 0.98 & 0.65 & 0.96 \\
\hline Kuru(2013) & 0.57 & Patient & 80 & 14 & 67 & 186 & 0.83 & 0.30 & 0.85 \\
\hline Numao(2013) & 0.45 & Patient & 136 & 57 & 101 & 56 & 0.64 & 0.71 & 0.70 \\
\hline Ibrahiem(2012) & 0.73 & Patient & 14 & 11 & 57 & 10 & 0.84 & 0.58 & 0.56 \\
\hline Portalez(2012) & 0.48 & Lesion & 404 & 47 & 34 & 47 & 0.42 & 0.90 & 0.89 \\
\hline Watanabe(2012) & 0.48 & Patient & 485 & 73 & 624 & 266 & 0.90 & 0.65 & 0.86 \\
\hline Haffner(2011) & 0.54 & Patient & 154 & 50 & 240 & 111 & 0.83 & 0.58 & 0.75 \\
\hline Rouse(2011) & 0.33 & Sextant & 145 & 11 & 74 & 72 & 0.87 & 0.67 & 0.92 \\
\hline Kitajima(2010) & 0.56 & Patient & 311 & 19 & 80 & 14 & 0.81 & 0.96 & 0.94 \\
\hline Labanaris(2010) & 0.73 & Patient & 17 & 73 & 96 & 74 & 0.57 & 0.19 & 0.18 \\
\hline Sciarra(2010) & 0.34 & Patient & 61 & 4 & 66 & 9 & 0.94 & 0.87 & 0.93 \\
\hline Kumar(2009) & 0.21 & Patient & 39 & 3 & 10 & 8 & 0.77 & 0.83 & 0.92 \\
\hline
\end{tabular}

high with a sensitivity of 0.87 and a specificity of 0.68 respectively.

Compared with the former review, the current study is the first meta-analysis to evaluate the performance of mpMRI based on prospective studies which could minimize the selection bias, besides, multiple subgroup analysis were performed in our study to explore the potential factors that may have an impact on the accuracy of mpMRI. Based on our results, pooled sensitivity was significantly higher in coil application group than in group without coils. Furthermore, the comparison between different magnetic strength suggested that field strength of MRI may also influence the accuracy of the diagnostic trial, the sensitivity from $3 \mathrm{~T}$ group is significantly higher than that from $1.5 \mathrm{~T}$ group. On the contrary, the comparison between biparametric MRI (bpMRI) (mpMRI without DCEI) and mutiparametric
MRI (mpMRI with DCEI) demonstrated similar point estimates for sensitivity and specificity. Through our subgroup analysis, the caregivers could offer the most suitable method of mpMRI for the pre-biopsy patients with suspected prostate cancer.

As the application of $3 \mathrm{~T}$ MRI became increasingly popular, mpMRI played an important role in the diagnostic of PCa. Murphy et al. [48] reported that increasing the magnetic field strength could improve the diagnostic accuracy of PCa. The study of Sertdemir et al. [49] suggested that prostate cancer could be better identified from prostatitis at $3 \mathrm{~T}$ strength field compared with 1.5 through DCEI. Theoretically, the MRI signal strength is proportional to the square of the static field strength while imaging noise increases drastically [50]. Barth et al. [51] indicated that the signal-noise ratio (SNR) of $3 \mathrm{~T}$ scanners is twice as good as $1.5 \mathrm{~T}$ in 


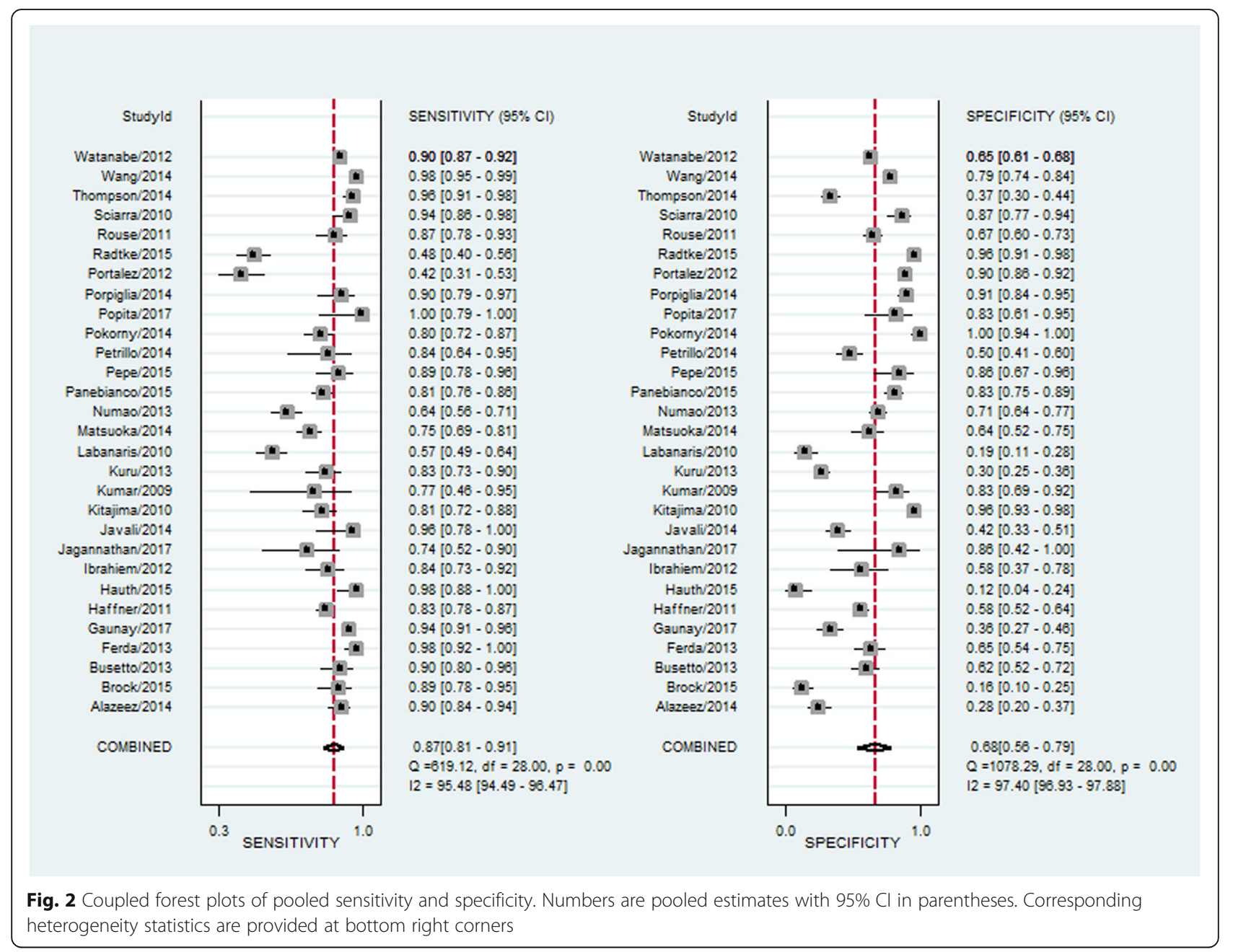

optimal condition. Several studies have also demonstrated the advantages of higher field strengths. However, the disadvantages and new challenges accompanied by higher field strength such as increased power deposition, artifacts related to susceptibility, and signal heterogeneity also should not be ignored. Methods to prevent those effects still require further exploration [52]. In addition, Nieuwenhove et al. [53] indicated that a fast MRI protocol (1.5 T magnet, T2 + DWI, < $15 \mathrm{~min}$ ) may replace the traditional $3.0 \mathrm{~T}$ mpMRI protocol safely without missing clinical significance so that the cost and contrast injection could be saved. Nevertheless, its conclusion was limited by a small number of endpoints. To validate these conclusions, a larger multicenter population-based trial is needed .

Although ERC has been applied to clinical practice since the early 1990s, its results have not been very heartening until the emergence of mpMR [54]. During the MRI examination, the ERC is inserted into rectal and clung tightly to the prostate to improve the image resolution and staging accuracy [55]. The application of the ERC may lead to higher costs because of material and longer examination time [56]. Moreover, many patients experienced discomfort feelings during the use of an ERC as well as the ensuing examination [57]. These disadvantages might limit the acceptance of ERC in mpMRI process. How to shorten the examination time and decrease the costs of imaging protocol are the problems researchers need to consider. Data of the study showed that ERC acceptance was directly related to how comfortable patients were. However, if such an examination was considered necessary, most patients would have been willing to undergo another examination with ERC and only $30 \%$ of patients will suffer from impaired diagnostic accuracy as a trade-off for higher patient comfort with the absence of ERC [58].

Limitations of mpMRI as an adjunct tool for prostate cancer screening are also noteworthy, such as increasing acquisition time, and the safety issue of gadolinium [59]. Recent studies have demonstrated high accuracy of bpMRI $[60,61]$. The analysis of the subgroup showed that bpMRI did not differ significantly from that of 


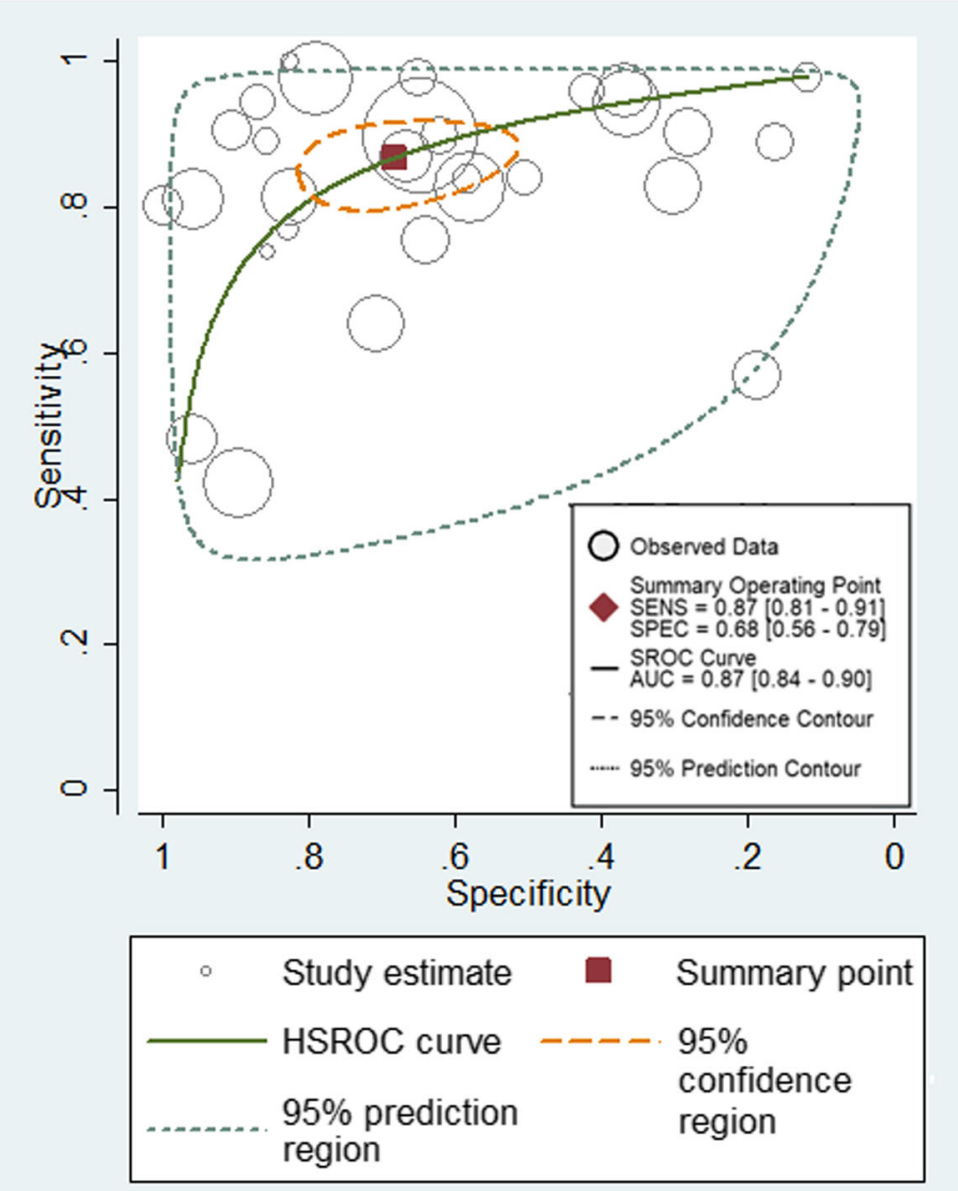

Fig. 3 Hierarchical summary receiver operating characteristic curve of the diagnostic performance of mpMRI for detecting prostate cancer

Table 3 Subgroup analysis of sensitivity and specificity according to the mpMRI

\begin{tabular}{|c|c|c|c|c|c|c|}
\hline parameter & category & nstudies & sensitivity & P1 & specificity & $\mathrm{P} 2$ \\
\hline \multirow[t]{2}{*}{ coil } & used & 9 & $0.89(0.83-0.89)$ & $<0.05$ & $0.67(0.46-0.88)$ & 0.69 \\
\hline & Not used & 16 & $0.86(0.80-0.92)$ & & $0.66(0.50-0.83)$ & \\
\hline \multirow[t]{2}{*}{ magnetic } & 3 & 10 & $0.88(0.81-0.95)$ & $<0.05$ & $0.77(0.61-0.93)$ & 0.99 \\
\hline & 1.5 & 17 & $0.85(0.78-0.91)$ & & $0.67(0.51-0.82)$ & \\
\hline \multirow[t]{2}{*}{ reference } & PI-RADS & 11 & $0.90(0.84-0.95)$ & 0.17 & $0.60(0.37-0.83)$ & 0.17 \\
\hline & others & 9 & $0.83(0.74-0.92)$ & & $0.78(0.61-0.96)$ & \\
\hline \multirow[t]{2}{*}{ MRI } & mutiparametric & 22 & $0.88(0.83-0.93)$ & 0.19 & $0.72(0.58-0.85)$ & 0.57 \\
\hline & biparametric & 7 & $0.82(0.70-0.93)$ & & $0.56(0.30-0.82)$ & \\
\hline \multirow[t]{2}{*}{ enrollment } & consecutive & 22 & $0.85(0.80-0.91)$ & $<0.05$ & $0.71(0.58-0.84)$ & 0.77 \\
\hline & Not consecutive & 7 & $0.91(0.84-0.98)$ & & $0.59(0.33-0.85)$ & \\
\hline \multirow[t]{2}{*}{ blinding } & blinded & 14 & $0.83(0.75-0.90)$ & $<0.05$ & $0.73(0.58-0.88)$ & 0.97 \\
\hline & Not mention & 15 & $0.90(0.85-0.95)$ & & $0.63(0.45-0.80)$ & \\
\hline \multirow[t]{2}{*}{ standard } & Low bias & 12 & $0.84(0.75-0.92)$ & $<0.05$ & $0.74(0.58-0.90)$ & 0.96 \\
\hline & High bias & 17 & $0.88(0.83-0.94)$ & & $0.64(0.48-0.90)$ & \\
\hline \multirow[t]{2}{*}{ Biopsy naive } & Yes & 9 & $0.87(0.80-0.95)$ & 0.35 & $0.64(0.40-0.88)$ & 0.28 \\
\hline & No & 7 & $0.81(0.70-0.92)$ & & $0.78(0.59-0.97)$ & \\
\hline
\end{tabular}



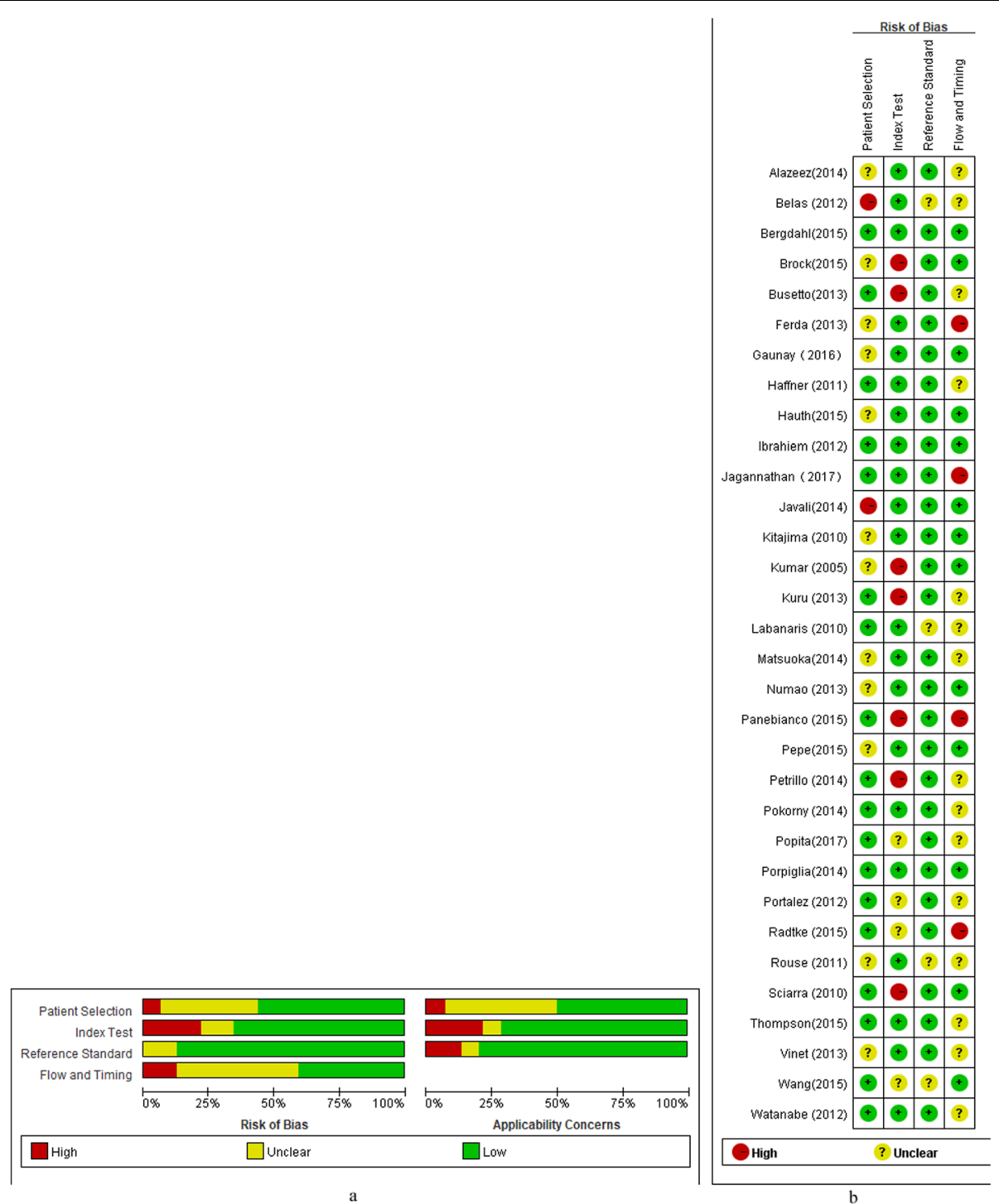

Fig. 4 (a)- Assessment of the risk of bias for included studies. (b)- Risk of bias summary graph

mpMRI which included an additional DCEI-MRI sequence. (Table 3) Currently, whether to include DCEIMRI in prostate MRI is a timely and controversial subject. Many articles focusing on bpMRI or comparisons between bpMRI and mpMRI were published [62, 63], and sessions focusing on this subject were being held at international congresses (i.e., European Association of Urology 2017, Korean Congress of Radiology 2017, and European Congress of Radiology 2018). In a study published in 2017, Greer et al. [64] suggested that the application of DCEI-MRI could benefit the diagnostic of prostate cancer because abnormal DCEI-MRI findings improve the cancer detection rate in each of the PIRADSv2 categories 2, 3, 4, and 5. However, those who advocated the use of bPMRI or opposed the use of DCEI-MRI suggested that compared with mpMRI,
bpMRI has several advantages such as shorter examination time, avoidance of risks associated with gadolinium-based contrast agents, and minimal risk of missing csPCa. Vargas et al. [65] found that DCEI-MRI helped to find only four additional tumors out of 152 patients. In addition, Kuhl et al. [61] demonstrated that the application of bpMRI protocol could reduce the MRI acquisition time from $34 \mathrm{~min} 19 \mathrm{~s}$ to $8 \mathrm{~min} 45 \mathrm{~s}$. However, most of the studies advocating bpMRI were retrospective studies with small number of patients. Larger- scale trials comparing bPMRI and mpMRI for further study was needed.

Our study also indicated that there was no statistically significance in both sensitivity and specificity between studies applied PI-RADS and studies with other criteria.The insignificance may owe to that multiple studies in 


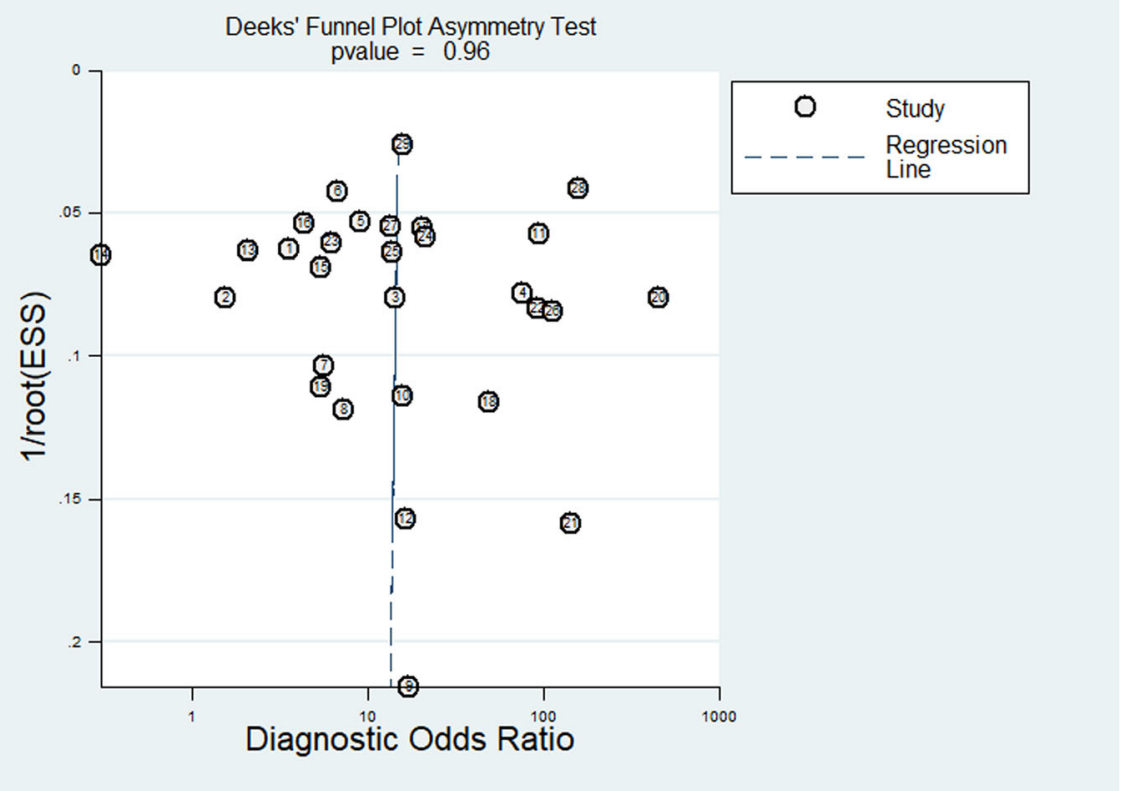

Fig. 5 Deeks' funnel plot. A $p$ value of 0.961 suggests that the likelihoodof publication bias is low.

PI-RADS group administrated PI-RADS version one as criteria. When we separated studies based on PI-RADS v2 from studies [21, 23, 25, 35] on old version and performed the meta-regression again, both sensitivity and specificity of PI-RADSv2 group were significantly higher than that from other group. The sensitivity of PIRADSv2 group was $0.89(0.81-0.97)$ and others was 0.88 (0.82-0.93) with $p=0.04$.

In order to standardize the acquisition, interpretation and reporting of prostate mpMRI, the European Society of Urogenital Radiology proposed the PI-RADS in 2012 [66]. In December 2014, the updated and simplified version (PI-RADSv2) was introduced to address the limitations and issues derived from the old version [67]. It summarized the level of likelihood of $\mathrm{PCa}$ in a five-point scale based on mpMRI findings considering the combination of T2WI, DWI, and DCE. One previous meta-analysis made a direct comparison between the two versions indicating that updated PI-RADSv2 showed significant improvement compared with the original PI-RADSv1 [68]. A more recent study which retrospectively investigated 166 patients after RP suggested PI-RADS v2 could be used to predict long-term outcomes following RP. PI-RADS could diminish variation in the interpretation and reporting of prostate imaging, especially among readers with varied experience levels [69]. Multiple studies has also proved mpMRI based on PI-RADS was useful in preventing unnecessary invasive procedures and helpful for the prediction and diagnosis of $\mathrm{PCa}$ and $\mathrm{csPCa}$ when combined with PSA and PSAD [70].
The administration of mpMRI for the evaluation of prostate cancer has increased drastically and this trend is likely to keep going because the technology is rapidly improving and its applications are expanding. A great number of previous studies have been focused on the accuracy of negative pre-biopsy prostate mpMRI in predicting a negative biopsy result for csPCa. However, the huge difference in csPCa definition prevented any explicit conclusions about mpMRI's ability to rule out aggressive cancer. Since biopsy results could not accurately reflect tumor burden and aggressiveness, how to define csPCa on biopsy appropriately is a complicated issue. Therefore, it is an urgent need to standardize the histological definition of csPCa in order to make comparisons among studies more meaningful.

The basic characteristic of imaging biomarkers is reproducibility as any biomarker fails its purpose, if it could not be reproducible or transfer to other patients, scanners or imaging protocols. Inter-observer variability is the reflection of reproducibility and is defined as the systematic differences among different observers [71]. Numerous acquisition techniques have been proposed in the past to solve the inter-observer variability in prostate cancer MRI. The occurence of quantitative MRI analysis has made it possible to compare between patients from different centers through quantitative hemodynamic parameters, and explore the change of biological characteristics before and after treatment. Instead of visualizing tissue characteristics indirectly through weighted contrasts, quantitative MRI attempts to directly measure 
them. Quantitative measurement of apparent diffusion coefficient (ADC) values has shown impressive diagnostic performance in discriminating csPCa from insignificant PCa, with overall AUC 0.880, and sensitivity of $71 \%$ and specificity of $88 \%$ and it has been proved these results are reproductive with high inter-observer correlation among different radiologists [72]. Although DCE played a minor role in the diagnosis of $\mathrm{PCa}$, it could describe the suspicious lesions in quantitative and semiquantitative measures which were different from DWI and T2 W1. Daniel et al. [73] indicated that combined with individualized T1-time correction, DCE could achieve excellent reproducibility, both intra- and interobserver variability were found to be increased.

Magnetic resonance fingerprinting (MRF) is a newly invented nuclear magnetic resonance parameter mapping method proposed by $\mathrm{Ma}$ et al. [74] which could estimate several quantitative tissue property parameters like $\mathrm{T} 1$ and $\mathrm{T} 2$ relaxation times simultaneously through transient signal evolutions and data analysis. With this ability, MRF could provide a solution to the problem of obtaining quantitative measures in an efficient manner and in short scanning times. Previous studies have demonstrated high reproducibility of MR fingerprinting parameter maps in solid tissues in the supratentorial region of the human brain [75]. Whether it could also improve the reproducibility and repeatability in $\mathrm{PCa}$ detection still requires the support of larger multicenter, randomized control trials. Moreover, 3D $1 \mathrm{H}-\mathrm{MRSI}$ of the prostate has also been proved by Lagemaat et al. [76] as an reproducible technique, however that conclusion was drawn based on small sample which limited its reliability; the larger population-based studies are also needed to prove that conclusion in the future.

\section{Limitations}

I. Included studies were heterogeneous in their methods, which may have an impact on the general applicability of the summary results. On the other hand, the methodologic variability can provide a lot of information for our subgroup analysis so that we can improve the diagnostic accuracy of mpMRI by identifying those factors in the future. II. Until recently, the definition of clinically relevant $\mathrm{PCa}$ varied considerably between each study. Therefore, we did not explore the possibility whether the prostate biopsy could be avoided through pre-biopsy MRI. III. Patients considered to be positive (signs and symptoms of prostate cancer) are more likely to get the gold standard, and only patients who get the gold standard are included in the study. This would result in falsely decreasing specificity and increasing sensitivity. It highlighted further areas of research that could help in defining the best use of mpMRI in the early detection of aggressive prostate cancer in the future. IV. Finally, data in some included studies are not provided completely, so we have to calculate the sensitivity and specificity by ourselves, this may affect the overall result of our study to some extent.

\section{Conclusion}

Although mpMRI can detect prostate cancer with excellent sensitivity, four main issues must be addressed before it becomes a triage test of prostate biopsy. I. The definition of the csPCa must be explicit so that further studies can be carried out to select the patients, the biopsy of whom can be obviated. II. The coil, the mpMRI with a stronger magnetic field, were recommended to improve the diagnostic accuracy of prostate cancer. III. The application of DCEI in the diagnostic of prostate cancer still needs to be testified. IV. Although efforts to standardize mpMRI technical protocols and interpretation have been made over the past few years, it is still urgent to improve mpMRI specificity and interreader reproducibility.

\section{Abbreviations}

bpMRI: Biparametric MRI; Bx: Biopsy; csPCa: Clinically significant cancer;

DCEl: Dynamic contrast-enhanced imaging; DOR: Diagnostic odds ratio; DWI: Diffusion-weighted imaging; ERC: Endorectal coil; FB: First biopsy;

FN: False negative; FP: False positive; HSROC: Hierarchical summary receiver operating characteristic curve; IQR: Interquartile range;

mpMRI: Multiparametric magnetic resonance imaging; MRSI: Magnetic resonance spectroscopic imaging; NPV: Negative predictive value; NR: Not reported; PCa: Prostate cancer; PNB: Previous negative biopsy; PPV: Positive predictive value; RoB: Risk of bias; ROC: Receiver operating characteristic curve; RP: Radical prostatectomy; SNR: Signal-noise ratio; T2: Weighted imaging; TBx: Targeted biopsy; TN: True negative; TP: True positive; TPBX: Transperineal template biopsy; TRUS SBX: Transrectal ultrasound-guided standard biopsy

\section{Acknowledgements}

Not applicable.

\section{Authors' contributions}

ZL and RH:Data analysis,project development, Manuscript writing. XL and JK:Data collection, project development, Manuscript editing. YC and XW: Data analysis, Manuscript editing. YY and NO: Data collection, Project

development. YX and WZ and YX:Data analysis. YS: Acquired the data. All authors read and approved the final manuscript.

Funding

Not applicable.

Availability of data and materials

All data is included in the article and additional fles.

Ethics approval and consent to participate

Not applicable.

Consent for publication

Not applicable.

Competing interests

The authors declare that they have no competing interests.

\section{Author details}

'Department of Urology, Tianjin Medical University General Hospital, Tianjin 300211, People's Republic of China. ${ }^{2}$ Department of Urology, Second Hospital of Tianjin Medical University, Tianjin, China. 
Received: 11 January 2019 Accepted: 4 December 2019

Published online: 23 December 2019

\section{References}

1. Todua F, Gagua R, Maglakelidze M, Maglakelidze D. Cancer incidence and mortality - Major patterns in GLOBOCAN 2012, worldwide and Georgia. Bulletin of the Georgian National Academy of Science. 2015;9(1):169-173.

2. Haas GP, Delongchamps N, Brawley OW, Wang CY, de la Roza G. The worldwide epidemiology of prostate Cancer: perspectives from autopsy studies. Can J Urol. 2008;15(1):3866-71.

3. Pokorny MR, de Rooij M, Duncan E, Schroder FH, Parkinson R, Barentsz JO, Thompson LC. Prospective study of diagnostic accuracy comparing prostate cancer detection by transrectal ultrasound-guided biopsy versus magnetic resonance (MR) imaging with subsequent MR-guided biopsy in men without previous prostate biopsies. Eur Urol. 2014;66(1):22-9.

4. Stephenson S, Chang E, Marks L. Screening and detection advances in magnetic resonance image-guided prostate biopsy. The Urologic Clinics of North America. 2014;41:315-26.

5. Turkbey B, Albert PS, Kurdziel K, Choyke PL. Imaging localized prostate cancer: current approaches and new developments. AJR Am J Roentgenol. 2009;192(6):1471-80.

6. Rosenkrantz AB, Scionti SM, Mendrinos S, Taneja SS. Role of MRI in minimally invasive focal ablative therapy for prostate cancer. AJR Am J Roentgenol. 2011;197(1):W90-6.

7. Yerram NK, Volkin D, Turkbey B, Nix J, Hoang AN, Vourganti S, Gupta GN, Linehan WM, Choyke PL, Wood BJ, et al. Low suspicion lesions on multiparametric magnetic resonance imaging predict for the absence of high-risk prostate cancer. BJU Int. 2012;110(11 Pt B):E783-8.

8. Hamoen EHJ, de Rooij M, Witjes JA, Barentsz JO, Rovers MM. Use of the prostate imaging reporting and data system (PI-RADS) for prostate Cancer detection with multiparametric magnetic resonance imaging: a diagnostic meta-analysis. Eur Urol. 2015;67(6):1112-21.

9. Moldovan PC, Van den Broeck T, Sylvester R, Marconi L, Bellmunt J, van den Bergh RCN, Bolla M, Briers E, Cumberbatch MG, Fossati N, et al. What is the negative predictive value of multiparametric magnetic resonance imaging in excluding prostate Cancer at biopsy? A systematic review and metaanalysis from the European Association of Urology prostate Cancer guidelines panel. Eur Urol. 2017;72(2):250-66.

10. Kim JY, Kim SH, Kim YH, Lee HJ, Kim MJ, Choi MS. Low-risk prostate cancer: the accuracy of multiparametric MR imaging for detection. Radiology. 2014; 271(2):435-44.

11. Sartor AO, Hricak H, Wheeler TM, Coleman J, Penson DF, Carroll PR, Rubin MA, Scardino PT. Evaluating localized prostate cancer and identifying candidates for focal therapy. Urology. 2008;72(6 Suppl):S12-24.

12. Dominguez C, Plata M, Catano JG, Palau M, Aguirre D, Narvaez J, Trujillo S, Gomez F, Trujillo CG, Caicedo Jl, et al. Diagnostic accuracy of multiparametric magnetic resonance imaging in detecting extracapsular extension in intermediate and high - risk prostate cancer. Int Braz J Urol. 2018;44(4):688-96.

13. Lamb BW, Tan WS, Rehman A, Nessa A, Cohen D, O'Neil J, Green JS, Hines JE. Is Prebiopsy MRI good enough to avoid prostate biopsy? A cohort study over a 1-year period. Clin Genitourin Cancer. 2015;13(6):512-7.

14. Schoots IG. Omission of systematic transrectal ultrasound guided biopsy from the MRI targeted approach in men with previous negative prostate biopsy might still be premature. Ann Transl Med. 2016;4(10):205.

15. Pepe P, Garufi A, Priolo G, Pennisi M. Can 3-tesla pelvic phased-array multiparametric MRI avoid unnecessary repeat prostate biopsy in patients with PSA < 10 ng/mL? Clin Genitourin Cancer. 2015;13(1):e27-30.

16. Aydin H, Hekimogl B, Tatar IG. Limitations, disabilities, and pitfalls of dynamic contrast-enhanced MRl as a diagnostic modality in prostate cancer. AJR Am J Roentgenol. 2013;200(3):W326.

17. McInnes MDF, Moher D, Thombs BD, McGrath TA, Bossuyt PM, Clifford T, Cohen JF, Deeks JJ, Gatsonis C, Hooft L, et al. Preferred reporting items for a systematic review and meta-analysis of diagnostic test accuracy studies: the PRISMA-DTA statement. Jama. 2018;319(4):388-96.

18. Reitsma JB, Glas AS, Rutjes AW, Scholten RJ, Bossuyt PM, Zwinderman AH. Bivariate analysis of sensitivity and specificity produces informative summary measures in diagnostic reviews. J Clin Epidemiol. 2005;58(10):982-90.

19. Deeks JJ, Macaskill P, Irwig L. The performance of tests of publication bias and other sample size effects in systematic reviews of diagnostic test accuracy was assessed. J Clin Epidemiol. 2005;58(9):882-93.
20. Jagannathan $\mathrm{D}$, Indiran V. Accuracy of diffusion weighted images and $\mathrm{mr}$ spectroscopy in prostate lesions - our experience with endorectal coil on 1. 5 T MRI. J Clin Diagn Res: JCDR. 2017;11(5):Tc10-tc14.

21. Popita C, Popita AR, Sitar-Taut A, Petrut B, Fetica B, Coman I. 1.5-Tesla Multiparametric-Magnetic Resonance Imaging for the detection of clinically significant prostate cancer. Clujul Med (1957). 2017;90(1):40-8.

22. Gaunay G, Patel V, Shah P, Moreira D, Hall SJ, Vira MA, Schwartz M, Kreshover J, Ben-Levi E, Villani R, et al. Role of multi-parametric MRI of the prostate for screening and staging: experience with over 1500 cases. Asian J Urol. 2017;4(1):68-74.

23. Wang $X$, Wang JY, Li CM, Zhang YQ, Wang JL, Wan B, Zhang W, Chen M, Li SY, Wan G, et al. Evaluation of the prostate imaging reporting and data system for magnetic resonance imaging diagnosis of prostate Cancer in patients with prostate-specific antigen $<20 \mathrm{ng} / \mathrm{ml}$. Chin Med J. 2016;129(12):1432-8.

24. Brock $M$, von Bodman $C$, Jüri $P$, Wolfgang $B$, Philipp $M S$, Joachim N. Detecting Prostate Cancer A Prospective Comparison of Systematic Prostate Biopsy With Targeted Biopsy Guided by Fused MRI and Transrectal Ultrasound. Medicine. 2015;112:605-610.

25. Hauth $\mathrm{E}$, Hohmuth $\mathrm{H}$, Cozub-Poetica C, Bernand S, Beer M, Jaeger $\mathrm{H}$. Multiparametric MRI of the prostate with three functional techniques in patients with PSA elevation before initial TRUS-guided biopsy. Br J Radiol. 2015:88(1054):20150422.

26. Panebianco V, Barchetti F, Sciarra A, Ciardi A, Indino EL, Papalia R, Gallucci M, Tombolini V, Gentile V, Catalano C. Multiparametric magnetic resonance imaging vs. standard care in men being evaluated for prostate cancer: a randomized study. Urol Oncol. 2015;33(1):17.e11-7.

27. Radtke JP, Kuru TH, Boxler S, Alt CD, Popeneciu IV, Huettenbrink C, Klein T, Steinemann S, Bergstraesser C, Roethke M, et al. Comparative analysis of transperineal template saturation prostate biopsy versus magnetic resonance imaging targeted biopsy with magnetic resonance imagingultrasound fusion guidance. J Urol. 2015;193(1):87-94.

28. Thompson JE, Moses D, Shnier R, Brenner P, Delprado W, Ponsky L, Pulbrook M, Bohm M, Haynes AM, Hayen A, et al. Multiparametric magnetic resonance imaging guided diagnostic biopsy detects significant prostate cancer and could reduce unnecessary biopsies and over detection: a prospective study. J Urol. 2014;192(1):67-74.

29. Abd-Alazeez M, Kirkham A, Ahmed HU, Arya M, Anastasiadis E, Charman SC, Freeman A, Emberton M. Performance of multiparametric MRI in men at risk of prostate cancer before the first biopsy: a paired validating cohort study using template prostate mapping biopsies as the reference standard. Prostate Cancer Prostatic Dis. 2014;17(1):40-6.

30. Javali TD, Dwivedi DK, Kumar R, Jagannathan NR, Thulkar S, Dinda AK. Magnetic resonance spectroscopy imaging-directed transrectal ultrasound biopsy increases prostate cancer detection in men with prostate-specific antigen between 4-10 $\mathrm{ng} / \mathrm{mL}$ and normal digital rectal examination. Int J Urol. 2014;21(3):257-62.

31. Matsuoka Y, Numao N, Saito K, Tanaka H, Kumagai J, Yoshida S, Koga F, Masuda H, Kawakami S, Fujii Y, et al. Combination of diffusion-weighted magnetic resonance imaging and extended prostate biopsy predicts lobes without significant cancer: application in patient selection for hemiablative focal therapy. Eur Urol. 2014;65(1):186-92.

32. Petrillo A, Fusco R, Setola SV, Ronza FM, Granata V, Petrillo M, Carone G, Sansone M, Franco R, Fulciniti F, et al. Multiparametric MRI for prostate cancer detection: performance in patients with prostate-specific antigen values between 2.5 and $10 \mathrm{ng} / \mathrm{mL}$. J Magn Reson Imaging : JMRI. 2014; 39(5):1206-12.

33. Porpiglia F, Russo F, Manfredi M, Mele F, Fiori C, Bollito E, Papotti M, Molineris I, Passera R, Regge D. The roles of multiparametric magnetic resonance imaging, PCA3 and prostate health index-which is the best predictor of prostate cancer after a negative biopsy? J Urol. 2014;192(1): 60-6.

34. Busetto GM, De Berardinis E, Sciarra A, Panebianco V, Giovannone R, Rosato S, D'Errigo P, Di Silverio F, Gentile V, Salciccia S. Prostate cancer gene 3 and multiparametric magnetic resonance can reduce unnecessary biopsies: decision curve analysis to evaluate predictive models. Urology. 2013;82(6): 1355-60.

35. Ferda J, Kastner J, Hora M, Hes O, Finek J, Topolcan O, Kreuzberg B. A role of multifactorial evaluation of prostatic 3T MRI in patients with elevated prostatic-specific antigen levels: prospective comparison with ultrasoundguided transrectal biopsy. Anticancer Res. 2013;33(6):2791-5. 
36. Kuru TH, Roethke MC, Seidenader J, Simpfendorfer T, Boxler S, Alammar K, Rieker P, Popeneciu VI, Roth W, Pahernik S, et al. Critical evaluation of magnetic resonance imaging targeted, transrectal ultrasound guided transperineal fusion biopsy for detection of prostate cancer. J Urol. 2013; 190(4):1380-6.

37. Numao N, Yoshida S, Komai Y, Ishii C, Kagawa M, Kijima T, Yokoyama M, Ishioka J, Matsuoka Y, Koga F, et al. Usefulness of pre-biopsy multiparametric magnetic resonance imaging and clinical variables to reduce initial prostate biopsy in men with suspected clinically localized prostate cancer. J Urol. 2013;190(2):502-8.

38. Ibrahiem El, Mohsen T, Nabeeh AM, Osman Y, Hekal IA, Abou El-Ghar M. DWI-MRI: single, informative, and noninvasive technique for prostate cancer diagnosis. ScientificWorldJournal. 2012;2012:973450.

39. Portalez D, Mozer P, Cornud F, Renard-Penna R, Misrai V, Thoulouzan M, Malavaud B. Validation of the European Society of Urogenital Radiology scoring system for prostate cancer diagnosis on multiparametric magnetic resonance imaging in a cohort of repeat biopsy patients. Eur Urol. 2012; 62(6):986-96.

40. Watanabe $Y$, Terai A, Araki T, Nagayama M, Okumura A, Amoh Y, Ishimori T, Ishibashi M, Nakashita S, Dodo Y. Detection and localization of prostate cancer with the targeted biopsy strategy based on ADC map: a prospective large-scale cohort study. Journal of magnetic resonance imaging : JMRI. 2012;35(6):1414-21.

41. Haffner J, Lemaitre L, Puech P, Haber GP, Leroy X, Jones JS, Villers A. Role of magnetic resonance imaging before initial biopsy: comparison of magnetic resonance imaging-targeted and systematic biopsy for significant prostate cancer detection. BJU Int. 2011;108(8 Pt 2):E171-8.

42. Rouse P, Shaw G, Ahmed HU, Freeman A, Allen C, Emberton M. Multiparametric magnetic resonance imaging to rule-in and rule-out clinically important prostate cancer in men at risk: a cohort study. Urol Int. 2011;87(1): 49-53.

43. Kitajima K, Kaji Y, Fukabori Y, Yoshida K, Suganuma N, Sugimura K. Prostate cancer detection with 3 T MRI: comparison of diffusion-weighted imaging and dynamic contrast-enhanced MRI in combination with T2-weighted imaging. J Magn Reson Imaging : JMRI. 2010;31(3):625-31.

44. Labanaris AP, Engelhard K, Zugor V, Nutzel R, Kuhn R. Prostate cancer detection using an extended prostate biopsy schema in combination with additional targeted cores from suspicious images in conventional and functional endorectal magnetic resonance imaging of the prostate. Prostate Cancer Prostatic Dis. 2010;13(1):65-70.

45. Sciarra A, Panebianco V, Ciccariello M, Salciccia S, Cattarino S, Lisi D, Gentilucci A, Alfarone A, Bernardo S, Passariello R, et al. Value of magnetic resonance spectroscopy imaging and dynamic contrast-enhanced imaging for detecting prostate cancer foci in men with prior negative biopsy. Clin Cancer Res. 2010;16(6):1875-83

46. Kumar V, Jagannathan NR, Kumar R, Thulkar S, Gupta SD, Hemal AK, Gupta NP. Transrectal ultrasound-guided biopsy of prostate voxels identified as suspicious of malignancy on three-dimensional (1) H MR spectroscopic imaging in patients with abnormal digital rectal examination or raised prostate specific antigen level of 4-10 ng/ml. NMR Biomed. 2007;20(1):11-20.

47. Porpiglia F, Manfredi M, Mele F, Cossu M, Bollito E, Veltri A, Cirillo S, Regge $D$, Faletti R, Passera R, et al. Diagnostic pathway with multiparametric magnetic resonance imaging versus standard pathway: results from a randomized prospective study in biopsy-naive patients with suspected prostate Cancer. Eur Urol. 2017;72(2):282-8.

48. Murphy G, Haider M, Ghai S, Sreeharsha B. The expanding role of MRI in prostate cancer. AJR Am J Roentgenol. 2013;201(6):1229-38.

49. Sertdemir M, Schoenberg SO, Sourbron S, Hausmann D, Heinzelbecker J, Michaely HJ, Dinter DJ, Weidner AM. Interscanner comparison of dynamic contrast-enhanced MRI in prostate cancer: 1.5 versus 3 T MRI. Investig Radiol. 2013;48(2):92-7.

50. Soher BJ, Dale BM, Merkle EM. A review of MR physics: 3T versus 1.5T. Magn Reson Imaging Clin N Am. 2007;15(3):277-90 v.

51. Barth MM, Smith MP, Pedrosa I, Lenkinski RE, Rofsky NM. Body MR imaging at $3.0 \mathrm{~T}$ : understanding the opportunities and challenges. Radiographics. 2007:27(5):1445-62 discussion 1462-1444.

52. Mazaheri $Y$, Vargas HA, Nyman G, Akin O, Hricak H. Image artifacts on prostate diffusion-weighted magnetic resonance imaging: trade-offs at 1.5 tesla and 3.0 tesla. Acad Radiol. 2013;20(8):1041-7.

53. Van Nieuwenhove $S$, Saussez TP, Thiry S, Trefois P, Annet L, Michoux N, Lecouvet $F$, Tombal B. Prospective comparison of a fast 1.5T biparametric to the 3.0T multi-parametric ESUR magnetic resonance imaging protocol as triage test for men at risk of prostate cancer. BJU Int. 2018;9:123.

54. Hassanzadeh E, Glazer DI, Dunne RM, Fennessy FM, Harisinghani MG, Tempany CM. Prostate imaging reporting and data system version 2 (PIRADS V2): a pictorial review. Abdom Radiol (NY). 2017;42(1):278-89.

55. Hricak H, White S, Vigneron D, Kurhanewicz J, Kosco A, Levin D, Weiss J, Narayan P, Carroll PR. Carcinoma of the prostate gland: MR imaging with pelvic phased-array coils versus integrated endorectal--pelvic phased-array coils. Radiology. 1994;193(3):703-9.

56. Bomers JG, Barentsz JO. Standardization of multiparametric prostate MR imaging using PI-RADS. Biomed Res Int. 2014;2014:431680.

57. Dickinson L, Ahmed HU, Allen C, Barentsz JO, Carey B, Futterer JJ, Heijmink SW, Hoskin PJ, Kirkham A, Padhani AR, et al. Magnetic resonance imaging for the detection, localisation, and characterisation of prostate cancer: recommendations from a European consensus meeting. Eur Urol. 2011; 59(4):477-94.

58. Baur AD, Daqqaq T, Wagner M, Maxeiner A, Huppertz A, Renz D, Hamm B, Fischer T, Durmus T. T2- and diffusion-weighted magnetic resonance imaging at $3 \mathrm{~T}$ for the detection of prostate cancer with and without endorectal coil: an intraindividual comparison of image quality and diagnostic performance. Eur J Radiol. 2016;85(6):1075-84.

59. Stanzione A, Imbriaco M, Cocozza S, Fusco F, Rusconi G, Nappi C, Mirone V, Mangiapia F, Brunetti A, Ragozzino A, et al. Erratum to Biparametric 3t magnetic resonance imaging for prostatic cancer detection in a biopsy-naive patient population: A further improvement of PI-RADS v2? [Eur. J. Radiol. 85 (12) (2016) 2269-2274]. Eur J Radiol. 2017;87:125.

60. Woo S, Suh CH, Kim SY, Cho JY, Kim SH, Moon MH. Head-to-head comparison between Biparametric and multiparametric MRI for the diagnosis of prostate Cancer: a systematic review and meta-analysis. AJR Am J Roentgenol. 2018;211(5):W226-w241.

61. Kuhl CK, Bruhn R, Kramer N, Nebelung S, Heidenreich A, Schrading S. Abbreviated Biparametric prostate MR imaging in men with elevated prostate-specific antigen. Radiology. 2017;285(2):493-505.

62. Jambor I, Bostrom PJ, Taimen P, Syvanen K, Kahkonen E, Kallajoki M, Perez IM, Kauko T, Matomaki J, Ettala O, et al. Novel biparametric MRI and targeted biopsy improves risk stratification in men with a clinical suspicion of prostate cancer (IMPROD trial). J Magn Reson Imaging : JMRI. 2017;46(4): 1089-95.

63. Fascelli M, Rais-Bahrami S, Sankineni S, Brown AM, George AK, Ho R, Frye T, Kilchevsky A, Chelluri R, Abboud S, et al. Combined Biparametric prostate magnetic resonance imaging and prostate-specific antigen in the detection of prostate Cancer: a validation study in a biopsy-naive patient population. Urology. 2016;88:125-34.

64. Greer MD, Shih JH, Lay N, Barrett T, Kayat Bittencourt L, Borofsky S, Kabakus IM, Law YM, Marko J, Shebel H, et al. Validation of the dominant sequence paradigm and role of dynamic contrast-enhanced imaging in PI-RADS version 2. Radiology. 2017;285(3):859-69.

65. Vargas HA, Hotker AM, Goldman DA, Moskowitz CS, Gondo T, Matsumoto K, Ehdaie B, Woo S, Fine SW, Reuter VE, et al. Updated prostate imaging reporting and data system (PIRADS v2) recommendations for the detection of clinically significant prostate cancer using multiparametric MRI: critical evaluation using whole-mount pathology as standard of reference. Eur Radiol. 2016;26(6):1606-12.

66. Iu PP. ESUR prostate MR guidelines. Eur Radiol. 2013;23(8):2320-1.

67. Weinreb JC, Barentsz JO, Choyke PL, Cornud F, Haider MA, Macura KJ, Margolis D, Schnall MD, Shtern F, Tempany CM, et al. PI-RADS prostate imaging - reporting and data system: 2015, version 2. Eur Urol. 2016;69(1): 16-40.

68. Woo S, Suh CH, Kim SY, Cho JY, Kim SH. Diagnostic performance of prostate imaging reporting and data system version 2 for detection of prostate Cancer: a systematic review and diagnostic meta-analysis. Eur Urol. 2017; 72(2):177-88.

69. Kim R, Kim CK. Prognostic significance for long-term outcomes following radical prostatectomy in men with prostate cancer: evaluation with prostate imaging reporting and data system version 2. Korean Journal of Radiology. 2019;20(2):256-64

70. Liu C, Liu S-L, Wang Z-X, Yu K, Feng C-X, Ke Z, Wang L, Zeng X-Y. Using the prostate imaging reporting and data system version 2 (PI-RIDS v2) to detect prostate cancer can prevent unnecessary biopsies and invasive treatment. Asian J Androl. 2018;20(5):459-64. 
71. Steenbergen P, Haustermans K, Lerut E, Oyen R, De Wever L, Van den Bergh L, Kerkmeijer LG, Pameijer FA, Veldhuis WB, van der Voort van Zyp JR, et al. Prostate tumor delineation using multiparametric magnetic resonance imaging: inter-observer variability and pathology validation. Radiother Oncol. 2015;115(2):186-90.

72. Nguyen TB, Ushinsky A. Utility of quantitative apparent diffusion coefficient measurements and normalized apparent diffusion coefficient ratios in the diagnosis of clinically significant peripheral zone prostate cancer. European Journal of Radiology. 2018;91(1088):20180091.

73. Daniel M, Polanec SH, Wengert G, Clauser P, Pinker K, Helbich TH, Georg D, Baltzer PAT. Intra- and inter-observer variability in dependence of T1-time correction for common dynamic contrast enhanced MRI parameters in prostate cancer patients. Eur J Radiol. 2019;116:27-33.

74. Ma D, Gulani V, Seiberlich N, Liu K, Sunshine JL, Duerk JL, Griswold MA. Magnetic resonance fingerprinting. Nature. 2013;495(7440):187-92.

75. Korzdorfer G, Kirsch R. Reproducibility and Repeatability of MR Fingerprinting Relaxometry in the Human Brain. Journal of Magnetic Resonance Imaging. 2019;292(2):429-37.

76. Lagemaat MW, Zechmann CM, Futterer JJ, Weiland E, Lu J, Villeirs GM, Holshouser BA, van Hecke P, Lemort M, Schlemmer HP, et al. Reproducibility of 3D 1H MR spectroscopic imaging of the prostate at 1.5T. J Magn Res Imaging : JMRI. 2012;35(1):166-73.

\section{Publisher's Note}

Springer Nature remains neutral with regard to jurisdictional claims in published maps and institutional affiliations.

Ready to submit your research? Choose BMC and benefit from:

- fast, convenient online submission

- thorough peer review by experienced researchers in your field

- rapid publication on acceptance

- support for research data, including large and complex data types

- gold Open Access which fosters wider collaboration and increased citations

- maximum visibility for your research: over $100 \mathrm{M}$ website views per year

At BMC, research is always in progress.

Learn more biomedcentral.com/submissions 